\title{
Evaluation of Taurine by HPTLC Reveals the Mask of Adulterated Edible Bird's Nest
}

\author{
Peishan Teo, Fucui Ma, and Daicheng Liu \\ Key Laboratory of Animal Resistance, College of Life Science, Shandong Normal University, 88 East Wenhua Road, \\ Jinan 250014, China
}

Correspondence should be addressed to Daicheng Liu; liudch@sdnu.edu.cn

Received 27 May 2013; Accepted 17 July 2013

Academic Editor: Lian-Wen Qi

Copyright (C) 2013 Peishan Teo et al. This is an open access article distributed under the Creative Commons Attribution License, which permits unrestricted use, distribution, and reproduction in any medium, provided the original work is properly cited.

\begin{abstract}
Detection of amino acid is an effective and common method to determine adulteration in edible bird's nest. Therefore, a simple and sensitive method was developed to detect taurine for determining adulteration in edible bird's nest in the future. Sample was separated on precoated silica gel GF254 high-performance thin layer chromatographic plates. Separation of taurine was performed by $\mathrm{n}$-propyl alcohol : ethanol: water : glacial acetic acid $(5.2: 0.8: 2: 2, \mathrm{v}: \mathrm{v}: \mathrm{v}: \mathrm{v})$. Densitometric analysis of taurine was carried out in the absorbance mode at $485 \mathrm{~nm}$. The method was validated for precision, intra- and interday variation, and recovery. This study proved that high-performance thin layer chromatography is a simple, rapid, precise, and selective method for qualitative and quantitative analysis of taurine in edible bird's nest.
\end{abstract}

\section{Introduction}

Edible bird's nest is the nest built by swiftlets. It is an expensive traditional Chinese medicine and nutraceutical food which has high medicinal value. Edible bird's nest can enhance immunologic function, dissolve phlegm, suppress cough, alleviate asthma, cure tuberculosis, improve the voice, and can also be used in antiaging, anticancer, curing stomach ulcers, haematemesis, general debility, and asthenia, and increase energy and metabolism, and so forth [1-3]. Therefore, different kinds of materials are added into the edible bird's nest by illegal vendors. Such materials as Tremella fungus, karaya gum, fried pork skin, soybean, red seaweed, egg white, gelatin, rice, milk, starch, agar, and fish vesicae, are incorporated into edible bird's nest. Other adulteration methods include staining, bleaching, and incorporating cheaper edible bird's nest into the more expensive ones [2, 4].

Based on the previous reports, edible bird's nest contains many chemical compounds, including lipid (0.14\%-1.28\%), ash $(2.1 \%)$, carbohydrate $(25.62 \%-27.26 \%)$, and protein $(62 \%$ $63 \%)$. The amino acids found in edible bird's nest are aspartic acid (Asp), threonine (Thr), serine (Ser), glutamic acid (Glu), proline (Pro), glycine (Gly), alanine (Ala), valine (Val), methionine (Met), isoleucine (Ile), leucine (Leu), tyrosine
(Tyr), phenylalanine (Phe), histidine (His), lysine (Lys), $\mathrm{NH}_{3}$, tryptophan (Trp), and arginine (Arg) [2, 4-8]. However, taurine was not detected in edible bird's nest. It can become an aiding method to recognize adulterated edible bird's nest. Simultaneous determination of several amino acids plays an important role to disclose adulterated edible bird's nest in the future.

Many authentication methods have been confirmed, that is, empirical measures, composition analysis, optical microscope, scanning electron microscopy (SEM), fluorescence method, modified xanthoproteic nitric acid test, gas chromatography (GC), capillary GC, infrared spectrometry (IR) and Fourier transform infrared spectroscopy (FTIR), ultraviolet spectroscopy, spectrophotometric method, SDSPAGE, isoelectrofocusing (IEF), two-dimensional gel electrophoresis (2-DE), atomic absorption, and molecular biological technology [2, 4, 9]. Detection of amino acids is an effective, simple, fast, and economic method compared with the authentication methods mentioned above.

Taurine (2-aminoethanesulphonic acid) is a free $\beta$-amino acid containing sulfur and is widely distributed in biological fluids and tissues, such as milk, urine, and muscle tissue. It plays important roles in many physiological functions and various diseases such as antioxidant [10], psychosis [11, 12], 
cancer [13], protection for liver [14], sepsis [15], and retinitis pigmentosa [16]. Thus, the detection of taurine could provide useful information for us to understand the healthy food, hepatology, and disorders.

To our knowledge, no article related to the stability HPTLC when detecting taurine in edible bird's nest has been reported in the current literature. Therefore, the aim of this study was to provide simple and rapid extraction, quantification, qualitative, and new authentication methods of taurine in edible bird's nest.

\section{Experimentals}

2.1. Chemicals and Materials. Unprocessed white edible bird's nest was obtained from CINRA Food Industries SDN. BHD (Malaysia). The bird's nests were processed as described by Guo et al., 2006 [17]. The grounded nest samples were used in the following experiments. Taurine standard (T0625, $\geq 99 \%$ lyophilized powder) was purchased from Sigma Chemical Co. (St. Louis, MO, USA). All chemicals including solvents were of analytical grade. Precoated silica gel GF254 HPTLC plates $\left(10 \times 10 \mathrm{~cm}^{2}\right)$ were purchased from Haiyang Chemical Co. (Qingdao, Shandong, China). Triketohydrindene hydrate was purchased from Merck Chemical Co. (Darmstadt, Germany).

2.2. Instrumentation. A Camag (Muttenz, Switzerland) thin layer chromatography scanner 3 with Wincats 1.4 .1 software, a development chamber consists of a twin trough glass chamber, an ultrasonic cleaner bath KQ-500 DB (Kunshan Ultrasonic Instrument Ltd., Suzhou, China), Buchi rotating evaporation instrument (Flawil, Switzerland), air oven, Eppendorf high-speed centrifuge (Hamburg, Germany) were used.

2.3. Chromatographic Conditions. The parameters were as follows: test plate: precoated silica gel GF254 HPTLC plates; format: $10 \times 10 \mathrm{~cm}^{2}$; development chamber: twin trough glass chamber $10 \times 12 \times 5 \mathrm{~cm}^{3}$; spotting volume of sample: $10 \mu \mathrm{L}$; mobile phase: $\mathrm{n}$-propyl alcohol : ethanol : water : glacial acetic acid (5.2: $0.8: 2: 2, \mathrm{v}: \mathrm{v}: \mathrm{v}: \mathrm{v})$; chamber saturation time: $20 \mathrm{~min}$ (mobile phase); room temperature: $23 \pm 2$ degrees Celsius; relative humidity: $55 \pm 3 \%$. Migration distance: $60 \mathrm{~mm}$; migration time: $40 \mathrm{~min}$; scanning speed: $20 \mathrm{~mm} / \mathrm{s}$; data resolution: $50 \mu \mathrm{m}$ step; reflectance-absorbance wavelength: $485 \mathrm{~nm}$; analyzing software: Wincats 1.4.1.

2.4. Extraction Method. The sample and water $(1: 9, \mathrm{v}: \mathrm{v})$ were mixed in a $50 \mathrm{~mL}$ centrifuge tube, and the tube was placed into an ultrasonic cleaner bath $(40 \mathrm{MHz}$, at room temperature). Each sample was sonicated three times with each for $20 \mathrm{~min}$. After sonication, the supernatant was obtained by centrifugation at $9000 \mathrm{rpm}$ for $10 \mathrm{~min}$ and then mixed with ethanol $(3: 7, \mathrm{v}: \mathrm{v})$ in the light resistant container for $30 \mathrm{~min}$. Then, the extract was centrifuged at $9000 \mathrm{rpm}$ for $10 \mathrm{~min}$. The supernatant was concentrated by rotating evaporation to less than $1 \mathrm{~mL}$.
2.5. Chromogenic Method. $1 \mathrm{~g}$ of triketohydrindene hydrate was dissolved in $100 \mathrm{ml}$ ethanol. Subsequent to the development, HPTLC plates were dried in fuming cupboard for $5 \mathrm{~min}$. Then, triketohydrindene hydrate solution was sprayed onto the HPTLC plates, and the plates were dried in the air oven (110 degrees Celsius) for $5 \mathrm{~min}$. Densitometric scanning was performed on Camag TLC scanner 3 at the reflectanceabsorbance wavelength of $485 \mathrm{~nm}$ and analyzed by Wincats 1.4.1.

2.6. Standard Solution Preparation. The standard solution was prepared by accurately weighed taurine dissolved in distilled water. The concentration of taurine was $0.12,0.192$, $0.24,0.36$, and $0.48 \mathrm{mg} / \mathrm{mL}$, respectively and $1 \mu \mathrm{L}$ of every concentration was spotted on the HPTLC plates. Quantitative concentration of each area was plotted with linear regression by TLC scanner.

2.7. Precision. Precision was determined by spotting 4 points of sample solution $(0.24 \mathrm{mg} / \mathrm{mL})$ on the same plate with each point $1 \mu \mathrm{L}$, and the plate was scanned on $0 \mathrm{hr}, 2$ hours, 4 hours, 6 hours, and 8 hours.

2.8. Intra- and Interday Variation. To ensure precision of the method, intra- and interday variation studies were carried out at $0.24 \mu \mathrm{g}, 0.36 \mu \mathrm{g}$, and $0.48 \mu \mathrm{g}$. The concentrations chosen fell in the linearity range including the highest to middle points of the calibration curve. Sample solutions were analyzed every 12 hours for three days.

2.9. Recovery Studies. The sample solution was spiked with extra $50 \%, 100 \%$, and $150 \%$ of the standard taurine and the mixtures were analyzed by the previous method.

2.10. Limit of Detection and Limit of Quantitation. The limit of detection (LOD) and limit of quantitation (LOQ) were determined following the same method. The signal to noise ratio was determined. LOD was considered as 3.3:1 and LOQ as $10: 1$. They were calculated using the equation:

$$
\begin{aligned}
& \mathrm{LOD}=3.3 \times \frac{\mathrm{SD}}{S} \\
& \mathrm{LOQ}=10 \times \frac{\mathrm{SD}}{S},
\end{aligned}
$$

where SD is the standard deviation of the intercept and $S$ is the slope of the calibration plot.

2.11. Statistical Analysis. Statistical analysis was carried out with SPSS 13.0 for Windows (SPSS, Inc., Chicago, IL, USA). Analysis of variance was performed. 


\section{Results and Discussion}

3.1. Identification of Taurine. Four spots can be observed in the same horizontal line in Figure 1 (Rf value of $0.62 \pm 0.02$ ). This result proved that the sample and standard were of the same polarity. As is shown in Figure 2, the absorption wavelength curves of the sample and standard were parallel with each other in the all spectrodensitometric analysis graph. These results proved that the sample and standard were of the same compound and of the same purity. The optimal absorption wavelength obtained was $485 \mathrm{~nm}$ from Figure 2. The above results proved the existence of taurine in edible bird's nest. Based on the previous reports, derivatization reagent for HPTLC with postchromatographic derivatization is triketohydrindene hydrate. The optimal absorption wavelength after derivatization was reported to be $490 \mathrm{~nm}$ [18] and $525 \mathrm{~nm}$ [19]. However, the optimal wavelength was found to be $485 \mathrm{~nm}$ in the present work. Different solvents which triketohydrindene hydrate was dissolved in might be a reason of optimal wavelength difference.

3.2. Extraction Method. The ultrasonically assisted extraction is a quick and effective method because the ultrasounds produce cell disruption and particle size reduction and compound can be dissolved perfectly in solvents $[19,20]$. In the present extraction method impurities were removed by ethanol, and the effect of the impurities on the detection of taurine was reduced. Triketohydrindene hydrate is commonly used to stain amino acids. Thus, it is a very important technique using HPTLC to qualify and quantify taurine.

3.3. Spot Stability. In the precision experiments, there was no significant difference among the peak area value between 0 , $2,4,6$, and $8 \mathrm{hrs}$. As was shown in Table 1 , the spotting was stable and the RSD was $0.83 \%$ after 4 hours. Table 2 presented that the RSD of the intra- and interday was $0.88 \%-0.78 \%$ and $0.75 \%-1.17 \%$, respectively which could indicate that the robustness of this method was acceptable. Average recovery of taurine was $99.68 \%$ as listed in Table 3 . The LOD was found to be $16 \mathrm{ng}$. The LOQ was $49 \mathrm{ng}$. The linearity range was $120-$ $480 \mathrm{ng}$. The above analysis of the data proved the repeatability and stability of this method.

3.4. HPTLC and Total Taurine Content. At present, numerous methods developed for measuring taurine have been reported which include gas chromatography (GC) [21], gas chromatography-mass spectrometry (GC-MS) [22], highperformance liquid chromatography (HPLC) [23, 24], capillary electrophoresis (CE), [25], and high-performance thin layer chromatography (HPTLC) $[18,19]$. In the layer chromatographic analysis, various kinds of solvent systems have been used, for example, chloroform-ethanol-acetic acidacetone-water $(54: 27: 10: 2: 2, \mathrm{v}: \mathrm{v}: \mathrm{v}: \mathrm{v}: \mathrm{v})$ and $\mathrm{n}$-butanolglacial acetic acid-96\% ethanol-water $(4: 2: 3: 3, \mathrm{v}: \mathrm{v}: \mathrm{v}: \mathrm{v})$. Taurine in energy drinks and syrup preparations was determined by HPTLC in previous study $[18,19]$. Thus, our present work will modify the method in the previous studies. For example, we provided new extraction method

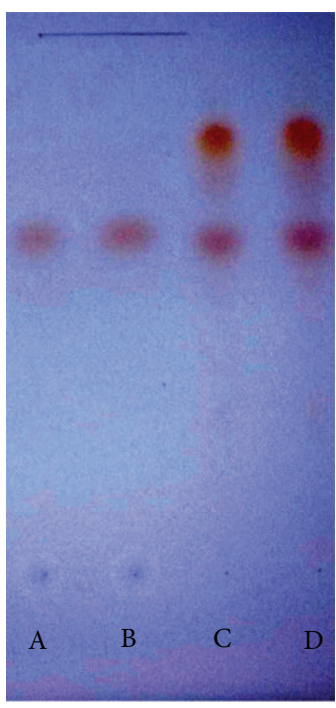

Figure 1: HPTLC plates of taurine. A, B: standard; C, D: samples.

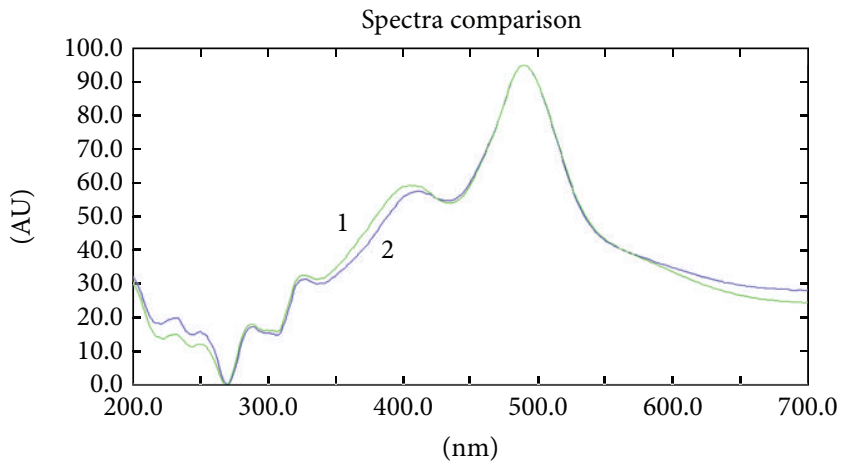

FIGURE 2: Spectrodensitometric analysis. 1: standard; 2: samples.

and modified development system in this experiment. The sample resources in the previous and present experiments are different. In analytical techniques, there is a two-step derivatization procedure converting taurine into volatile derivatives in the GC and GC-MS methods. In addition, the $\mathrm{CE}$ method requires a complex adjustment for analysis of taurine [24]. In the HPLC method, several samples and standard cannot be run simultaneously in the same mobile phase. HPTLC method is widely used mainly due to the purpose of environmental friendly concept and reducing the use of solvent. Since several samples can be analyzed simultaneously using a small quantity of mobile phase, the analytical time and cost of per analysis can be reduced. It was proved to be an efficient, simple, and precise method for quantitative analysis of taurine in present work. Moreover, this method is a useful qualitative method to detect taurine. Linear regression equation of taurine was $Y=4234.5154+$ $20.6178 X$ ( $Y$ is the response while $X$ is the amount of taurine) and $r=0.99928, \mathrm{RSD}=1.87 \%$. Total content of taurine was $12.62 \mu \mathrm{g} / \mathrm{g}$ in edible bird's nest. Statistical data analysis proved that this HPTLC method is precise, specific, accurate, and stable for the determination of taurine in edible bird's nest. 
TABle 1: Precision.

\begin{tabular}{|c|c|c|c|c|c|}
\hline Amount ( $\mu \mathrm{g} / \mathrm{spot})$ & Time (hrs) & Peak area values & $\begin{array}{c}\text { Mean } \\
(4-6 \mathrm{hrs})\end{array}$ & $\begin{array}{c}\text { SD } \\
(4-6 \mathrm{hrs})\end{array}$ & $\begin{array}{c}\text { RSD \% } \\
(4-6 \mathrm{hrs})\end{array}$ \\
\hline \multirow{5}{*}{$0.24 \mu \mathrm{g}$} & 0 & 18422.5 & \multirow{5}{*}{9306.25} & \multirow{5}{*}{77.38} & \multirow{5}{*}{0.83} \\
\hline & 2 & 14274 & & & \\
\hline & 4 & 9289.75 & & & \\
\hline & 6 & 9287.75 & & & \\
\hline & 8 & 9341.25 & & & \\
\hline
\end{tabular}

TABLE 2: Intra- and interday variation.

\begin{tabular}{|c|c|c|c|c|c|c|}
\hline \multirow{2}{*}{ Amount ( $\mu \mathrm{g} / \mathrm{spot})$} & \multicolumn{3}{|c|}{ Intraday persion } & \multicolumn{3}{|c|}{ Interday persion } \\
\hline & Peak area values (Mean) & SD & RSD \% & Peak area values (Mean) & SD & RSD \% \\
\hline 0.24 & 9411.58 & 82.82 & 0.88 & 9439.00 & 113.68 & 1.20 \\
\hline 0.36 & 11724.58 & 96.24 & 0.82 & 11730.78 & 107.62 & 0.92 \\
\hline 0.48 & 14695.17 & 114.98 & 0.78 & 14705.78 & 123.02 & 0.84 \\
\hline
\end{tabular}

TABle 3: Recovery.

\begin{tabular}{lccc}
\hline Level \% & Observed \% & Recovery \% & Average recovery \% \\
\hline 50 & 49.63 & 99.25 & \\
100 & 99.93 & 99.93 & 99.68 \\
150 & 149.78 & 99.85 & \\
\hline
\end{tabular}

Taurine is mainly found in animal tissues and is found in millimolar concentrations [26]. Our present experiment indicated that taurine in edible bird's nest is in micromolar concentrations. The distinguished differences of taurine concentration in plant, animals, and edible bird's nest make the determination of taurine to be a simple and effective method to detect adulteration in edible bird's nest. So far, no method can be used solely to authenticate edible bird's nest. Two or several authentication methods are often used together to complete the work. Taurine detection can be a simple and sensitive aiding method to recognize adulterated edible bird's nest.

\section{Conclusions}

The proposed analytical HPTLC method is precise, specific, accurate, and stable. This method may be applied in routine analysis of taurine in edible bird's nest, and it may be a useful method to determine adulteration.

\section{Authors' Contribution}

Peishan Teo and Fucui Ma contributed equally to this work.

\section{Disclaimer}

The authors declare that none of the authors of this paper has a direct financial relation with the commercial identities mentioned in their paper.

\section{Conflict of Interests}

There is no conflict of interests.

\section{References}

[1] J. J. Hobbs, "Problems in the harvest of edible birds' nests in Sarawak and Sabah, Malaysian Borneo," Biodiversity and Conservation, vol. 13, no. 12, pp. 2209-2226, 2004.

[2] F. Ma and D. Liu, "Sketch of the edible bird's nest and its important bioactivities," Food Research International, vol. 48, pp. 559-567, 2012.

[3] Y. Wu, Y. Chen, B. Wang et al., "Application of SYBRgreen PCR and 2DGE methods to authenticate edible bird's nest food," Food Research International, vol. 43, no. 8, pp. 2020-2026, 2010.

[4] M. F. Marcone, "Characterization of the edible bird's nest the "Caviar of the East"', Food Research International, vol. 38, no. 10, pp. 1125-1134, 2005.

[5] R. H. Kathan and D. I. Weeks, "Structure studies of Collocalia mucoid. I. Carbohydrate and amino acid composition," Archives of Biochemistry and Biophysics, vol. 134, no. 2, pp. 572-576, 1969.

[6] H. Nakagawa, Y. Hama, T. Sumi et al., "Occurrence of a nonsulfated chondroitin proteoglycan in the dried saliva of Collocalia swiftlets (edible bird's-nest)," Glycobiology, vol. 17, no. 2, pp. 157-164, 2007.

[7] M. Oda, S. Ohta, T. Suga, and T. Aoki, "Study on food components: the structure of $\mathrm{N}$-linked asialo carbohydrate from the edible bird's nest built by Collocalia fuciphaga," Journal of Agricultural and Food Chemistry, vol. 46, no. 8, pp. 30473053, 1998.

[8] C. C. Wang, “The composition of Chinese edible birds' nests and the nature of their proteins," Journal of Biological Chemistry, vol. 49, pp. 429-439, 1921.

[9] Y. Q. Yu, L. Xue, H. Wang, H. X. Zhou, X. F. Zhu, and B. S. $\mathrm{Li}$, "Determination of edible bird's nest and its products by gas chromatography," Journal of Chromatographic Science, vol. 38, no. 1, pp. 27-32, 2000.

[10] J. G. Alvarez and B. T. Storey, "Taurine, hypotaurine, epinephrine and albumin inhibit lipid peroxidation in rabbit spermatozoa and protect against loss of motility," Biology of Reproduction, vol. 29, no. 3, pp. 548-555, 1983. 
[11] M. Jeevanandam, D. H. Young, L. Ramias, and W. R. Schiller, "Aminoaciduria of severe trauma," American Journal of Clinical Nutrition, vol. 49, no. 5, pp. 814-822, 1989.

[12] K. H. Tachiki, H. C. Hendrie, J. Kellams, and M. H. Aprison, "A rapid column chromatographic procedure for the routine measurement of taurine in plasma of normals and depressed patients," Clinica Chimica Acta, vol. 75, no. 3, pp. 455-465, 1977.

[13] G. E. Gray, A. M. Landel, and M. M. Meguid, "Taurinesupplemented total parenteral nutrition and taurine status of malnourished cancer patients," Nutrition, vol. 10, no. 1, pp. 1115, 1994.

[14] S. L. Devi and C. V. Anuradha, "Oxidative and nitrosative stress in experimental rat liver fibrosis: protective effect of taurine," Environmental Toxicology and Pharmacology, vol. 29, no. 2, pp. 104-110, 2010.

[15] L. I. Woolf, A. C. Groves, and J. P. Mooore, "Arterial plasma amino acids in patients with serious postoperative infection and in patients with major fractures," Surgery, vol. 79, no. 3, pp. 283292, 1976.

[16] S. M. Uma, M. Satapathy, and A. Sitaramayya, "Decreased plasma taurine levels in retinitis pigmentosa," Biochemical Medicine, vol. 30, no. 1, pp. 49-52, 1983.

[17] C. T. Guo, T. Takahashi, W. Bukawa et al., "Edible bird's nest extract inhibits influenza virus infection," Antiviral Research, vol. 70, no. 3, pp. 140-146, 2006.

[18] G. Indrayanto, T. K. Sia, and Y. I. Wibowo, "Densitometric determination of taurine and L-lysine hydrochloride in an energy drink and in multi-vitamin syrup, and validation of the method," Journal of Planar Chromatography, vol. 14, no. 1, pp. 24-27, 2001.

[19] M. Aranda and G. Morlock, "Simultaneous determination of riboflavin, pyridoxine, nicotinamide, caffeine and taurine in energy drinks by planar chromatography-multiple detection with confirmation by electrospray ionization mass spectrometry," Journal of Chromatography A, vol. 1131, no. 1-2, pp. 253-260, 2006.

[20] F. Ma and D. Liu, "Extraction and determination of hormones in the edible bird's nest," Asian Journal of Chemistry, vol. 24, pp. 117-120, 2012.

[21] H. Kataoka, K. Matsuba, and M. Makita, "Determination of taurine in whole blood and plasma by gas liquid chromatography," Yakugaku Zasshi, vol. 104, no. 10, pp. 1054-1058, 1984.

[22] L. Marks, F. Iglicki, B. Rakotoambinina, F. Thullier, and B. Messing, "A gas chromatographic/electron impact mass spectrometric method for the isolation and derivatization of plasma taurine for use in stable isotope tracer kinetic studies," Journal of Mass Spectrometry, vol. 30, no. 12, pp. 1687-1693, 1995.

[23] G. P. McMahon, R. O’Kennedy, and M. T. Kelly, "Highperformance liquid chromatographic determination of taurine in human plasma using pre-column extraction and derivatization," Journal of Pharmaceutical and Biomedical Analysis, vol. 14, no. 8-10, pp. 1287-1294, 1996.

[24] H. Inoue, K. Fukunaga, and Y. Tsuruta, "Determination of taurine in plasma by high-performance liquid chromatography using 4-(5,6-dimethoxy-2-phthalimidinyl)2-methoxyphenylsulfonyl chloride as a fluorescent labeling reagent," Analytical Biochemistry, vol. 319, no. 1, pp. 138-142, 2003.

[25] M. T. Kelly, H. Faber, and D. Perrett, "Determination of taurine in plasma by capillary zone eletrophoresis following derivatisation with fluorescamine," Electrophoresis, vol. 21, pp. 699-705, 2000.
[26] T. Bouckenooghe, C. Remacle, and B. Reusens, "Is taurine a functional nutrient?" Current Opinion in Clinical Nutrition and Metabolic Care, vol. 9, no. 6, pp. 728-733, 2006. 

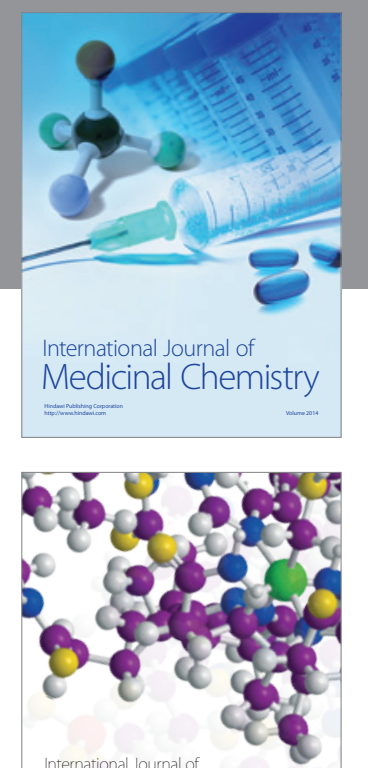

\section{Carbohydrate} Chemistry

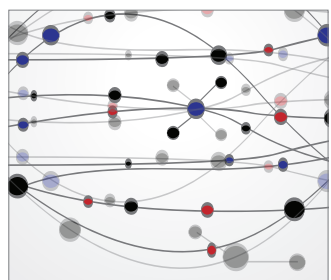

The Scientific World Journal
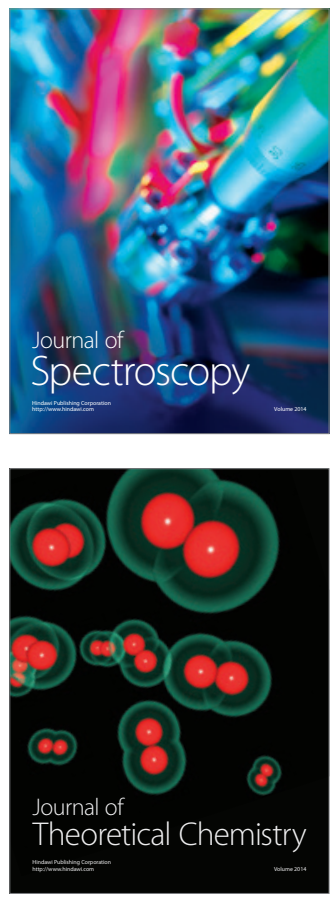
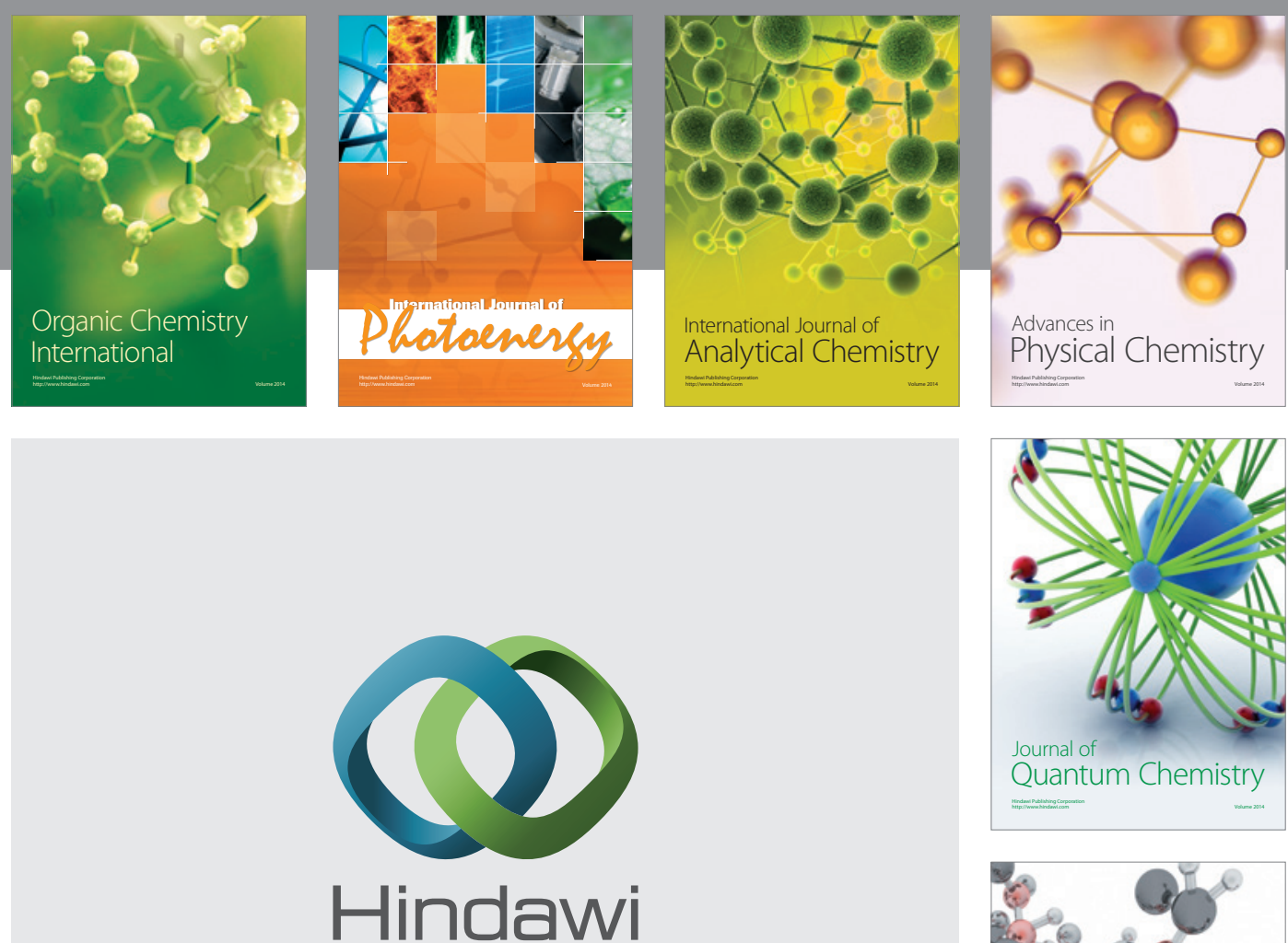

Submit your manuscripts at

http://www.hindawi.com

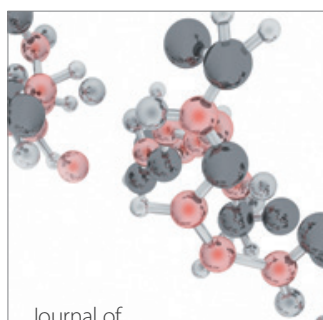

Analytical Methods

in Chemistry

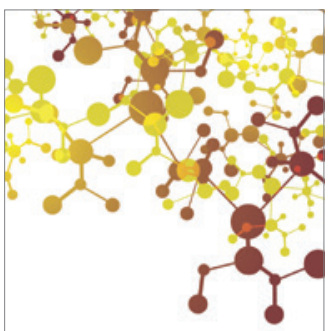

Journal of

Applied Chemistry

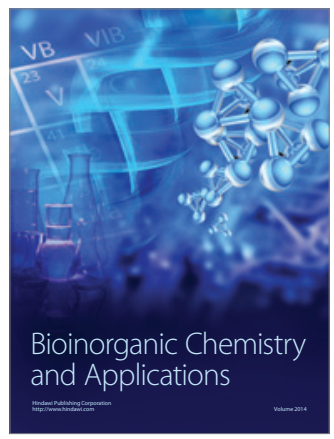

Inorganic Chemistry
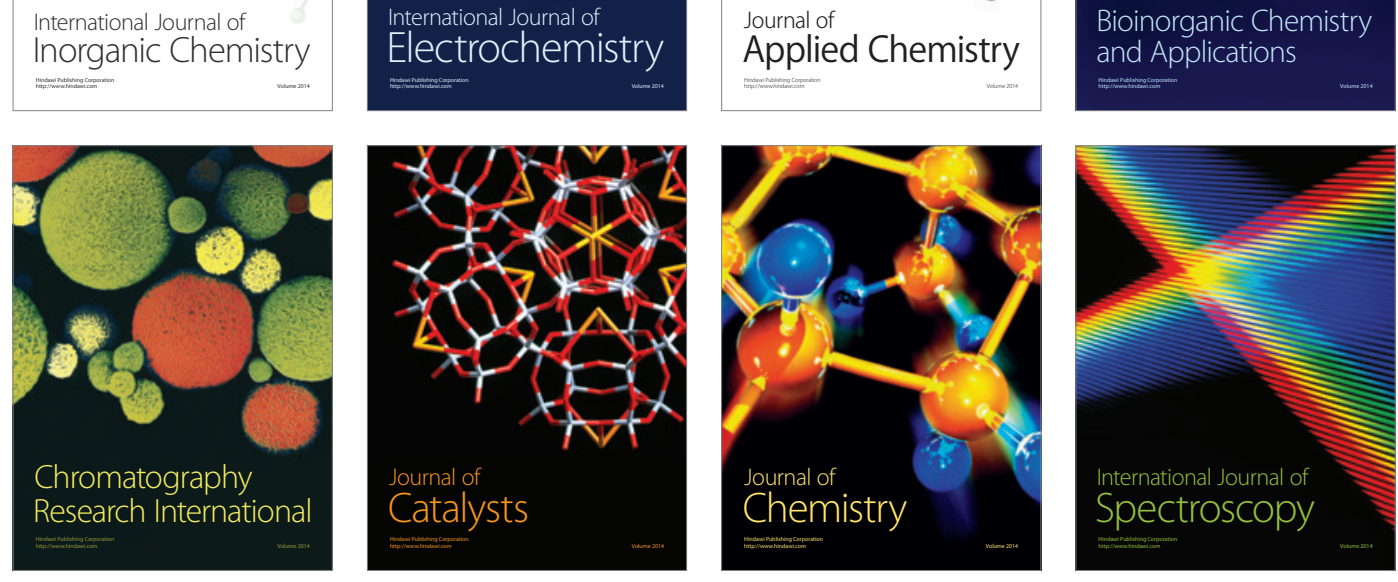